\title{
A 2-Component Laplace Mixture Model: Properties and Parametric Estimations ${ }^{i}$
}

\author{
Zakiah I. Kalantan ${ }^{1, *}$, Faten Alrewely ${ }^{2}$ \\ ${ }^{1}$ Faculyu of Science, King Abdulaziz University, Jeddah, Saudi Arabia \\ ${ }^{2}$ Faculty of Science, Al Jouf University, Sakaka, Saudi Arabia
}

Received July 1, 2019; Revised August 28, 2019; Accepted September 20, 2019

Copyright $\bigcirc 2019$ by authors, all rights reserved. Authors agree that this article remains permanently open access under the terms of the Creative Commons Attribution License 4.0 International License

\begin{abstract}
Mixture distributions have received considerable attention in life applications. This paper presents a finite Laplace mixture model with two components. We discuss the model properties and derive the parameters estimations using the method of moments and maximum likelihood estimation. We study the relationship between the parameters and the shape of the proposed distribution. The simulation study discusses the effectiveness of parameters estimations of Laplace mixture distribution.
\end{abstract}

Keywords Laplace Distribution, Mixture Distribution, Method of Moments, Maximum Likelihood Estimation

\section{Introduction}

Laplace distribution has wide applications in various fields such as engineering, business, medicine, and others. It is also known as a double exponential distribution because it is considered as two exponential distributions with additional location parameter. It considers as a member of lifetime distributions.

Mixture distributions and the problem of mixture decomposition about the identification of the constituent components and parameters dates back to 1846, but most of the reference is made due to the work of Karl Pearson in 1894 [7]. The approach taken by Pearson was to fit a univariate mixture of two normal to data through the choice of five parameters of the mixture in a way that empirical moments matched the model. The work by Pearson was successful in identifying two distinct sub-populations and also showing the flexibility of mixtures as a moment matching tool. Other later works focused on addressing the problems, but the invention of the modern computer and the popularization of the maximum likelihood parameterization techniques caused a stir in research work on mixture models [6]. In 2002, Figueiredo and Jain applied the finite mixture to unsupervised learning models and gave important insights into mixture models [3]. Bhowmick et al introduced the Laplace mixture model instead of the Gaussian mixture model due to the tail length and a weight of the Laplace distribution, then applied the mixture micro-experiments [2]. Ali and Nadarajah found the information matrices for the Gaussian distribution mixture and the Laplace distribution mixture [5]. A mixture of asymmetric Laplace and Gaussian distributions was estimated using the EM algorithm by Shenoy and Gorinevsky [11]. Amini-Seresht and Zhang provided random comparisons of two finite-mixture models with different mixing ratios and independent variables [1].

Most of the references presented comprehensive studies and applications of finite mixture models which is based on the work of McLachlan, and Peel [6]. Ramana et al., introduced the two-component mixture of Laplace and Laplace type bimodal distributions, and they find the properties and estimation for the common parameters between the two-component mixture [9]. The previous studies are based on equal mixing parameters or constant scale parameters. The aim of this paper is to study the two components Laplace mixture model and estimate its parameters in the case of unknown all model parameters using parametric estimation methods.

The paper is organized as follows: Section 2 presents the definition of Laplace mixture distribution. Section 3 discusses the distribution function. The properties of the proposed distribution are studied in Section 4. The estimation methods are presented in Section 5. The simulation studies are presented in Section 6. Finally, conclusions are drawn in Section 7.

\section{Laplace Mixture Distribution}

\subsection{Definition of Mixture Distribution}

The Consider dataset $X=\left(x_{1}, x_{2}, \ldots, x_{n}\right)$ be 
N-dimensional random variable, then $X$ follows $k$ components Laplace mixture distribution if its probability density function can be written as:

$$
f(x ; \underline{\theta})=\sum_{i=1}^{k} \alpha_{i} f_{i}\left(x ; \underline{\theta}_{i}\right)
$$

where $\alpha_{i}, i=1,2, \ldots, k$ are the mixing probabilities that satisfy $\alpha_{i} \geq 0$ and $\sum_{i=1}^{k} \alpha_{i}=1$.

\subsection{Mixture of a Two Laplace Distribution}

Let $X$ a random variable, the overall formula of the distribution of Laplace is

$$
f(x ; \theta)=\frac{1}{2 \lambda} \exp \left(-\frac{|x-\mu|}{\lambda}\right),
$$

where $-\infty<x<\infty,-\infty<\mu<\infty$, and $\lambda>0$.

In this paper, we will consider two components $(\mathrm{k}=2)$ for Laplace mixture (A2-CLPM) distribution, where the first component provides the proportion $\alpha_{1}$ and density parameters $\mu_{1}, \lambda_{1}$. The second one provides the proportion $\alpha_{2}=\left(1-\alpha_{1}\right)$ and the second density parameters $\mu_{2}, \lambda_{2}$, then the A2-CLPM distribution can be written as:

$$
\begin{gathered}
f(x ; \underline{\theta})= \\
\alpha_{1} \operatorname{Laplace}\left(\mu_{1}, \lambda_{1}\right)+\left(1-\alpha_{1}\right) \operatorname{Laplace}\left(\mu_{2}, \lambda_{2}\right)
\end{gathered}
$$

Then, the probability density function (pdf) for A2-CLPM distribution is

$f(x ; \underline{\theta})=\frac{\alpha_{1}}{2 \lambda_{1}} \exp \left(-\frac{\left|x-\mu_{1}\right|}{\lambda_{1}}\right)+\frac{\left(1-\alpha_{1}\right)}{2 \lambda_{2}} \exp \left(-\frac{\left|x-\mu_{2}\right|}{\lambda_{2}}\right)$,

where $-\infty<x<\infty,-\infty<\mu_{1}, \mu_{2}<\infty, \lambda_{1}, \lambda_{2}>0$, and $\alpha_{1}+\left(1-\alpha_{1}\right)=1$.

Figure 1 presents the curve of A2-CLPM distribution with parameters equal $\underline{\theta}=\left(\alpha_{1}, \mu_{1}, \mu_{2}, \lambda_{1}, \lambda_{2}\right)$.

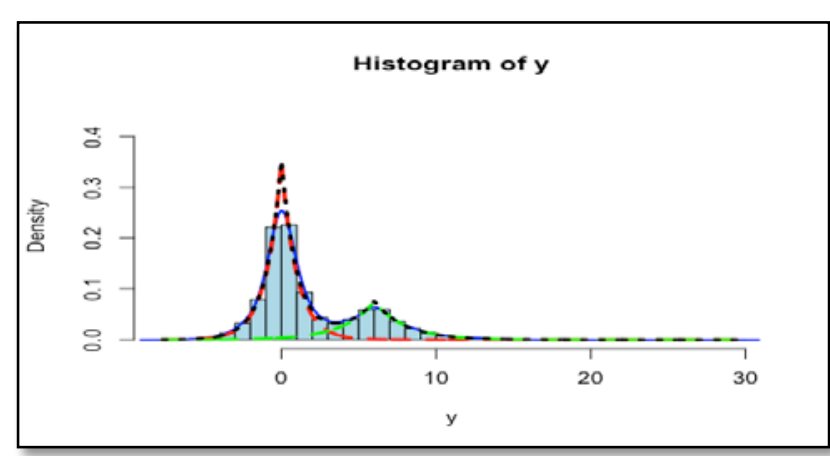

Figure 1. The frequency curves of the mixture Laplace distribution.

\section{The Cumulative Density Function (CDF)}

We state that the given pdf is a density function by computing the integral of the mixture distribution over its range, then we have

$$
\begin{gathered}
\int_{-\infty}^{+\infty} f(x ; \underline{\theta}) d x=\int_{-\infty}^{\infty}\left[\frac{\alpha_{1}}{2 \lambda_{1}} \exp \left(-\frac{\left|x-\mu_{1}\right|}{\lambda_{1}}\right)+\right. \\
\left.\frac{\left(1-\alpha_{1}\right)}{2 \lambda_{2}} \exp \left(-\frac{\left|x-\mu_{2}\right|}{\lambda_{2}}\right)\right] d x=1 .
\end{gathered}
$$

The cumulative distribution function (CDF) of $X$ defines as:

$$
F(x)=\int_{-\infty}^{x} f(w ; \underline{\theta}) d w
$$

$$
\begin{aligned}
F(x)=\int_{-\infty}^{x}\left[\frac{\alpha_{1}}{2 \lambda_{1}}\right. & \exp \left(-\frac{\left|w-\mu_{1}\right|}{\lambda_{1}}\right) \\
& \left.+\frac{\left(1-\alpha_{1}\right)}{2 \lambda_{2}} \exp \left(-\frac{\left|w-\mu_{2}\right|}{\lambda_{2}}\right)\right] d w
\end{aligned}
$$

then we have

$$
F(x)=\frac{1}{2}\left[\alpha_{1} \exp \left(\frac{x-\mu_{1}}{\lambda_{1}}\right)+\left(1-\alpha_{1}\right) \exp \left(\frac{x-\mu_{2}}{\lambda_{2}}\right)\right],
$$

for $x \leq \mu_{1}, \mu_{2}$

and

$$
\begin{gathered}
F(x)=\alpha_{1}\left[1-\frac{1}{2} \exp \left(-\frac{\left(x-\mu_{1}\right)}{\lambda_{1}}\right)\right] \\
+\left(1-\alpha_{1}\right)\left[1-\frac{1}{2} \exp \left(-\frac{\left(x-\mu_{2}\right)}{\lambda_{2}}\right)\right] \\
\quad \text { for } x>\mu_{1}, \mu_{2}
\end{gathered}
$$

\section{The Properties of Laplace Mixture Distribution}

In what follows, the distribution properties are studied by obtaining the mean, the mode, the median, and the variance of A2-CLPM distribution.

- The mean of the random variable $X$ is

$$
\begin{gathered}
\mu=E(x)=\int_{-\infty}^{+\infty} x f(x ; \underline{\theta}) d x \\
\mu= \\
\frac{\alpha_{1}}{2 \lambda_{1}} \int_{-\infty}^{+\infty} x \exp \left(-\frac{\left|x-\mu_{1}\right|}{\lambda_{1}}\right) d x+ \\
\frac{\left(1-\alpha_{1}\right)}{2 \lambda_{2}} \int_{-\infty}^{+\infty} x \exp \left(-\frac{\left|x-\mu_{2}\right|}{\lambda_{2}}\right) d x=\alpha_{1} \mu_{1}+\left(1-\alpha_{1}\right) \mu_{2} .
\end{gathered}
$$

- The mode of the random variable $X$ given from

$$
\frac{d}{d x} f(x ; \underline{\theta})=0 .
$$

when derived the equation (4) were $x \geq \mu$ or $x<\mu$, we get the same mode for both of them, the formula are following as:

$$
x=\frac{\lambda_{2} \mu_{1}+\lambda_{1} \mu_{2}}{\lambda_{1}+\lambda_{2}} .
$$

The median of this distribution can be obtained as

$$
p(x \leq c)=\frac{1}{2} \text {, }
$$


because of symmetry, we can suppose that

$$
c \leq \frac{\lambda_{2} \mu_{1}+\lambda_{1} \mu_{2}}{\lambda_{1}+\lambda_{2}} .
$$

- The variance and standard deviation of the mixture distribution.

The variance is defined as:

$$
\sigma^{2}=\operatorname{var}(x)=E\left(x^{2}\right)-(E(x))^{2} .
$$

Then, we find $E\left(x^{2}\right)$, as following as:

$$
\begin{gathered}
E\left(x^{2}\right)=\int_{-\infty}^{+\infty} x^{2} f(x ; \underline{\theta}) d x . \\
E\left(x^{2}\right)=\frac{\alpha_{1}}{2 \lambda_{1}} \int_{-\infty}^{+\infty} x^{2} \exp \left(-\frac{|x-\mu|}{\lambda_{1}}\right) d x+ \\
\frac{\left(1-\alpha_{1}\right)}{2 \lambda_{2}} \int_{-\infty}^{+\infty} x^{2} \exp \left(-\frac{|x-\mu|}{\lambda_{2}}\right) d x \\
E\left(x^{2}\right)=\alpha_{1}\left[\mu_{1}{ }^{2}+2 \lambda_{1}{ }^{2}\right]+\left(1-\alpha_{1}\right)\left[\mu_{2}{ }^{2}+2 \lambda_{2}{ }^{2}\right] .
\end{gathered}
$$

Then, substitute Eq (7) and Eq (13) in Eq (12), we get

$$
\begin{gathered}
\sigma^{2}=\operatorname{var}(x) \\
\sigma^{2}=\alpha_{1}\left[\mu_{1}{ }^{2}+2 \lambda_{1}{ }^{2}\right]+\left(1-\alpha_{1}\right)\left[\mu_{2}{ }^{2}+2 \lambda_{2}{ }^{2}\right]- \\
{\left[\alpha_{1} \mu_{1}+\left(1-\alpha_{1}\right) \mu_{2}\right]^{2} .}
\end{gathered}
$$

The stander deviation of the random variable $X$ is the square root of the variance.

$$
\begin{aligned}
& \sigma=\sqrt{\operatorname{var}(x)} \\
& \sigma=\sqrt{\alpha_{1}\left[\mu_{1}{ }^{2}+2 \lambda_{1}{ }^{2}\right]+\left(1-\alpha_{1}\right)\left[\mu_{2}{ }^{2}+2 \lambda_{2}{ }^{2}\right]}-\alpha_{1} \mu_{1}+ \\
& \left(1-\alpha_{1}\right) \mu_{2} \text {. }
\end{aligned}
$$

Tables (1-5) present the properties of three samples of size 1000 taken from Laplace mixture distribution, we compute the mean, mode, median, variance, skewness and kurtosis with different parameters values for Laplace mixture distribution. For simplicity, we take special cases of mixture distribution in order to study the performance of the Laplace mixture distribution.

In Table 2, we state the performance of scale parameters by assuming that location parameters $\mu_{1}=0, \mu_{2}=2, \alpha_{1}=0.3$ and $\lambda_{2}=2$. As expected, the simulation results illustrate that the Laplace mixture distribution has a positive skewness and a heavy tail which provides a good fitted model for life applications where outliers have located in the right tail of the mixture curve. As also as, the Laplace mixture distribution has a positive kurtosis equals 3.317 which indicates that the curve approximately has normal curve. For Table 2, we compute the distribution properties with different values of second scale parameter $\lambda_{2}$ by setting other parameters by $\mu_{1}=3, \mu_{2}=5, \alpha_{1}=0.4$ and $\lambda_{1}=0.5$.

The third case studies the properties of the proposed mixture model with different values of mixing parameter $\alpha_{1}=0.2,0.5$ and 0.9 , we assume $\mu_{1}=0, \mu_{2}=5, \lambda_{1}=$ 1 , and $\lambda_{2}=2$. The results are presented in Table 3, which illustrates equal values of mean and median. At $\alpha_{1}=0.2$, we observe that kurtosis equals 3.25 which indicates that the curve approximately has normal curve. In contrast, when $\alpha_{1}=0.5$ and 0.9 the curve has heavy tail. Table 4 displays the results of studying three samples from Laplace mixture distribution with parameters values $\alpha_{1}=0.6, \mu_{2}=3, \lambda_{1}=4$, and $\lambda_{2}=6$. In this case we use different values of first location parameter $\mu_{1}=(1,2,4)$. We observe that the three distributions have skewness $=$ 0.5 or 0.6 , and kurtosis $\cong 2.1$ which means that the curves are close to the normal distribution curve. The results of final case is presented in Table 5, it also draws the same conclusions that when $\mu_{1}=5, \quad \lambda_{1}=1, \lambda_{2}=5$ and $\alpha_{1}=0.2$, while the vector of second location number equals to $\mu_{2}=(1,2,3)$.

Table 1. The results of three samples of Laplace mixture distribution when $\lambda_{1}=(0.5,1,3)$

\begin{tabular}{|c|c|c|c|c|c|}
\hline$\left(\boldsymbol{\alpha}_{\mathbf{1}}, \boldsymbol{\mu}_{\mathbf{1}}, \boldsymbol{\mu}_{2}, \boldsymbol{\lambda}_{\mathbf{1}}, \boldsymbol{\lambda}_{\mathbf{2}}\right)$ & Mean & Median & Variance & Skew. & Kurt. \\
\hline$(\mathbf{0 . 3}, \mathbf{0}, \mathbf{2}, \mathbf{0 . 5}, \mathbf{2})$ & 0.0495 & 0.0144 & 0.0049 & 1.865 & 3.317 \\
\hline$(\mathbf{0 . 3}, \mathbf{0}, \mathbf{2}, \mathbf{1}, \mathbf{2})$ & 0.0494 & 0.0169 & 0.0038 & 1.212 & 0.005 \\
\hline$(\mathbf{0 . 3}, \mathbf{0}, \mathbf{2}, \mathbf{3}, \mathbf{2})$ & 0.0489 & 0.0253 & 0.0027 & 1.202 & 0.310 \\
\hline
\end{tabular}

Table 2. The results of three samples of Laplace mixture distribution when $\lambda_{2}=(1,2,3)$

\begin{tabular}{|c|c|c|c|c|c|}
\hline$\left(\boldsymbol{\alpha}_{\mathbf{1}}, \boldsymbol{\mu}_{\mathbf{1}}, \boldsymbol{\mu}_{\mathbf{2}}, \boldsymbol{\lambda}_{\mathbf{1}}, \boldsymbol{\lambda}_{\mathbf{2}}\right)$ & Mean & Median & Variance & Skew. & Kurt. \\
\hline$(\mathbf{0 . 4}, \mathbf{3}, \mathbf{5}, \mathbf{0 . 5}, \mathbf{1})$ & 0.0497 & 0.0023 & 0.0081 & 1.986 & 6.233 \\
\hline$(\mathbf{0 . 4}, \mathbf{3}, \mathbf{5}, \mathbf{0 . 5}, \mathbf{2})$ & 0.0486 & 0.0125 & 0.0062 & 2.442 & 0.733 \\
\hline$(\mathbf{0 . 4}, \mathbf{3}, \mathbf{5}, \mathbf{0 . 5}, \mathbf{3})$ & 0.0469 & 0.0191 & 0.0054 & 2.849 & 12.34 \\
\hline
\end{tabular}

Table 3. The results of three samples of Laplace mixture distribution when $\alpha_{1}=(0.2,0.5,0.9)$

\begin{tabular}{|c|c|c|c|c|c|}
\hline$\left(\boldsymbol{\alpha}_{\mathbf{1}}, \boldsymbol{\mu}_{\mathbf{1}}, \boldsymbol{\mu}_{\mathbf{2}}, \boldsymbol{\lambda}_{\mathbf{1}}, \boldsymbol{\lambda}_{\mathbf{2}}\right)$ & Mean & Median & Variance & Skew. & Kurt. \\
\hline$(\mathbf{0 . 2}, \mathbf{0}, \mathbf{5}, \mathbf{1}, \mathbf{2})$ & 0.0328 & 0.0328 & 0.0026 & 1.0183 & 3.259 \\
\hline$(\mathbf{0 . 5}, \mathbf{0}, \mathbf{5}, \mathbf{1}, \mathbf{2})$ & 0.0309 & 0.0309 & 0.0029 & 1.4448 & 5.078 \\
\hline$(\mathbf{0 . 9}, \mathbf{0}, \mathbf{5}, \mathbf{1}, \mathbf{2})$ & 0.0126 & 0.0126 & 0.0080 & 2.555 & 9.144 \\
\hline
\end{tabular}


Table 4. The results of three samples of Laplace mixture distribution when $\mu_{1}=(1,2,4)$

\begin{tabular}{|c|c|c|c|c|c|}
\hline$\left(\boldsymbol{\alpha}_{\mathbf{1}}, \boldsymbol{\mu}_{\mathbf{1}}, \boldsymbol{\mu}_{2}, \boldsymbol{\lambda}_{\mathbf{1}}, \boldsymbol{\lambda}_{\mathbf{2}}\right)$ & Mean & Median & Variance & Skew. & Kurt. \\
\hline$(\mathbf{0 . 6}, \mathbf{1}, \mathbf{3}, \mathbf{4}, \mathbf{6})$ & 0.0430 & 0.0364 & 0.0007 & 0.5553 & 2.082 \\
\hline$(\mathbf{0 . 6}, \mathbf{2}, \mathbf{3}, \mathbf{4}, \mathbf{6})$ & 0.0428 & 0.0360 & 0.0007 & 0.622 & 2.294 \\
\hline$(\mathbf{0 . 6}, \mathbf{4}, \mathbf{3}, \mathbf{4}, \mathbf{6})$ & 0.0418 & 0.0360 & 0.0008 & 0.5604 & 2.170 \\
\hline
\end{tabular}

Table 5. The results of three samples of Laplace mixture distribution when $\mu_{2}=(1,2,3)$

\begin{tabular}{|c|c|c|c|c|c|}
\hline$\left(\boldsymbol{\alpha}_{\mathbf{1}}, \boldsymbol{\mu}_{1}, \boldsymbol{\mu}_{\mathbf{2}}, \boldsymbol{\lambda}_{\mathbf{1}}, \boldsymbol{\lambda}_{\mathbf{2}}\right)$ & Mean & Median & Variance & Skew. & Kurt. \\
\hline$(\mathbf{0 . 7}, \mathbf{5}, \mathbf{1}, \mathbf{3}, \mathbf{1 . 5})$ & 0.0464 & 0.0346 & 0.002 & 0.4193 & 1.693 \\
\hline$(\mathbf{0 . 7}, \mathbf{5}, \mathbf{2}, \mathbf{3}, \mathbf{1 . 5})$ & 0.0464 & 0.0306 & 0.002 & 0.6367 & 1.912 \\
\hline$(\mathbf{0 . 7}, \mathbf{5}, \mathbf{3}, \mathbf{3}, \mathbf{1 . 5})$ & 0.0463 & 0.0273 & 0.002 & 0.9090 & 2.433 \\
\hline
\end{tabular}

\section{Parametric Estimation Methods}

In this section, we obtain the parameter estimates of Laplace mixture distribution using two parametric estimation methods: method of moments (MME) and maximum likelihood estimation (MLE) method. The illustrations are presented in the following subsections.

\subsection{The Method of Moments (MME)}

Let $X$ be a random variable with A2-CLPM $\left(\alpha_{1}, \mu_{1}, \mu_{2}, \lambda_{1}, \lambda_{2}\right)$ distribution. The $r^{\text {th }}$ moments are defined as:

$$
\begin{gathered}
E\left(x^{r}\right)=\int_{-\infty}^{+\infty} x^{r} f(x ; \underline{\theta}) d x \\
=\int_{-\infty}^{\infty} \boldsymbol{x}^{r}\left(\frac{\boldsymbol{\alpha}_{1}}{2 \lambda_{1}} \exp \left(-\frac{\left|x-\mu_{1}\right|}{\lambda_{1}}\right)+\frac{\left(1-\alpha_{1}\right)}{2 \lambda_{2}} \exp \left(-\frac{\left|x-\mu_{2}\right|}{\lambda_{2}}\right)\right) \boldsymbol{d} \boldsymbol{x} .
\end{gathered}
$$

For simplicity, we use the Taylor series instead of integration, because, we need to find eight moments, and solve these integrations are very hard, see [8], as following as:

$$
\begin{aligned}
& \grave{\mu_{r}}=E\left(x^{r}\right) \\
& =\left(\frac{\alpha_{1}}{2}\right) \sum_{k=0}^{r}\left[\frac{r !}{(r-k) !} \lambda_{1}{ }^{k} \mu_{1}{ }^{(r-k)}\left\{1+(-1)^{k}\right\}\right]+\left(\frac{\left(1-\alpha_{1}\right)}{2}\right) \sum_{k=0}^{r}\left[\frac{r !}{(r-k) !} \lambda_{2}{ }^{k} \mu_{2}{ }^{(r-k)}\left\{1+(-1)^{k}\right\}\right] . \\
& \mu_{r}=\left\{\begin{array}{c}
0 \quad, \text { if } r \text { is odd }, \\
2 \alpha_{1} \lambda_{1}{ }^{r} r !+2\left(1-\alpha_{1}\right) \lambda_{2}{ }^{r} r !, \text { if } r \text { is even. }
\end{array}\right.
\end{aligned}
$$

The $r^{\text {th }}$ means of the population are equal to

$$
M^{\prime}{ }_{r}=P_{1} \frac{\sum_{i=1}^{n} x_{i_{1}}{ }^{r}}{n_{1}}+\left(1-P_{1}\right) \frac{\sum_{i=1}^{n} x_{i_{2}}{ }^{r}}{n_{2}},
$$

where $r=1,2,3, \ldots, n$ and $P_{1}+\left(1-P_{1}\right)=1$ they are population mixing parameters.

Then, by solve equations, as following as:

$$
\grave{\mu_{r}}=M^{\prime}{ }_{r} .
$$

The estimations are made for the five parameters $\underline{\theta}=\left(\alpha_{1}, \mu_{1}, \mu_{2}, \lambda_{1}, \lambda_{2}\right)$. For this mixture distribution, we compute the first eight raw moments in order to obtain the moment estimations of distribution parameters. It is noted that, we find parameter estimation $\hat{\alpha}_{2}$ from compute $1-\hat{\alpha}_{1}$. Also, it is noted that $E\left(x^{r}\right)=0$, for odd values $>1$ of $r$ therefore, we base our estimation on the first moment and the even moments. Now, from Eq (17) we get the first moment, as following as: when $r=1$

$$
\grave{\mu_{1}}=E(x)=\alpha_{1} \mu_{1}+\left(1-\alpha_{1}\right) \mu_{2} .
$$

when $r=2$ 


$$
\dot{\mu}_{2}=E\left(x^{2}\right)=\alpha_{1}\left(\mu_{1}^{2}+2 \lambda_{1}^{2}\right)+\left(1-\alpha_{1}\right)\left(\mu_{2}^{2}+2 \lambda_{2}^{2}\right) .
$$

when $r=4$

$$
\dot{\mu}_{4}=E\left(x^{4}\right)=\alpha_{1}\left(\mu_{1}^{4}+12 \lambda_{1}^{2} \mu_{1}^{2}+24 \lambda_{1}^{4}\right)+\left(1-\alpha_{1}\right)\left(\mu_{2}^{4}+12 \lambda_{2}^{2} \mu_{2}^{2}+24 \lambda_{2}^{4}\right) .
$$

when $r=6$

$$
\begin{gathered}
\dot{\mu}_{6}=E\left(x^{6}\right)=\alpha_{1}\left(\mu_{1}{ }^{6}+30 \lambda_{1}{ }^{2} \mu_{1}{ }^{4}+360 \lambda_{1}{ }^{4} \mu_{1}{ }^{2}+720 \lambda_{1}{ }^{6}\right)+\left(1-\alpha_{1}\right) \\
\left(\mu_{2}{ }^{6}+30 \lambda_{2}{ }^{2} \mu_{2}{ }^{4}+360 \lambda_{2}{ }^{4} \mu_{2}{ }^{2}+720 \lambda_{2}{ }^{6}\right) .
\end{gathered}
$$

when $r=8$

$$
\begin{aligned}
\dot{\mu}_{8}= & E\left(x^{8}\right)=\alpha_{1}\left(\mu_{1}{ }^{8}+56 \lambda_{1}{ }^{2} \mu_{1}{ }^{6}+1680 \lambda_{1}{ }^{4} \mu_{1}{ }^{4}+20160 \lambda_{1}{ }^{6} \mu_{1}{ }^{2}+40320 \lambda_{1}{ }^{8}\right) \\
& +\left(1-\alpha_{1}\right)\left(\mu_{2}{ }^{8}+56 \lambda_{2}{ }^{2} \mu_{2}{ }^{6}+1680 \lambda_{2}{ }^{4} \mu_{2}{ }^{4}+20160 \lambda_{2}{ }^{6} \mu_{2}{ }^{2}+40320 \lambda_{2}{ }^{8}\right) .
\end{aligned}
$$

To obtain estimates of the distribution parameters, equate the five equations above with $\mathrm{Eq}(18)$ for $r=1,2,4,6,8$ and then solving them. As following as:

$$
\begin{aligned}
& \mu^{\prime}{ }_{1}=M_{1}^{\prime} \\
& \alpha_{1} \mu_{1}+\left(1-\alpha_{1}\right) \mu_{2}=P_{1} \frac{\sum_{i=1}^{n} x_{i_{1}}}{n_{1}}+\left(1-P_{1}\right) \frac{\sum_{i=1}^{n} x_{i_{2}}}{n_{2}} . \\
& \mu^{\prime}{ }_{2}=M_{2}^{\prime} \\
& \alpha_{1}\left(\mu_{1}^{2}+2 \lambda_{1}^{2}\right)+\left(1-\alpha_{1}\right)\left(\mu_{2}^{2}+2 \lambda_{2}^{2}\right) \\
& =P_{1} \frac{\sum_{i=1}^{n} x_{i_{1}}{ }^{2}}{n_{1}}+\left(1-P_{1}\right) \frac{\sum_{i=1}^{n} x_{i_{2}}{ }^{2}}{n_{2}} \text {. } \\
& \mu^{\prime}{ }_{4}=M^{\prime}{ }_{4} \\
& \alpha_{1}\left(\mu_{1}^{4}+12 \lambda_{1}^{2} \mu_{1}^{2}+24 \lambda_{1}^{4}\right)+\left(1-\alpha_{1}\right) \\
& \left(\mu_{2}{ }^{4}+12 \lambda_{2}{ }^{2} \mu_{2}{ }^{2}+24 \lambda_{2}{ }^{4}\right)=P_{1} \frac{\sum_{i=1}^{n} x_{i_{1}}{ }^{4}}{n_{1}}+\left(1-P_{1}\right) \frac{\sum_{i=1}^{n} x_{i_{2}}{ }^{4}}{n_{2}} \text {. } \\
& \mu^{\prime}{ }_{6}=M^{\prime}{ }_{6} \\
& \alpha_{1}\left(\mu_{1}{ }^{6}+30 \lambda_{1}{ }^{2} \mu_{1}{ }^{4}+360 \lambda_{1}{ }^{4} \mu_{1}{ }^{2}+720 \lambda_{1}{ }^{6}\right) \\
& +\left(1-\alpha_{1}\right)\left(\mu_{2}{ }^{6}+30 \lambda_{2}{ }^{2} \mu_{2}{ }^{4}+360 \lambda_{2}{ }^{4} \mu_{2}{ }^{2}+720 \lambda_{2}{ }^{6}\right) \\
& =P_{1} \frac{\sum_{i=1}^{n} x_{i_{1}}{ }^{6}}{n_{1}}+\left(1-P_{1}\right) \frac{\sum_{i=1}^{n} x_{i_{2}}{ }^{6}}{n_{2}} \text {. } \\
& \mu^{\prime}{ }_{8}=M^{\prime}{ }_{8} \\
& \alpha_{1}\left(\mu_{1}{ }^{8}+56 \lambda_{1}{ }^{2} \mu_{1}{ }^{6}+1680 \lambda_{1}{ }^{4} \mu_{1}{ }^{4}+20160 \lambda_{1}{ }^{6} \mu_{1}{ }^{2}+40320 \lambda_{1}^{8}\right)+ \\
& \left(1-\alpha_{1}\right)\left(\mu_{2}{ }^{8}+56 \lambda_{2}{ }^{2} \mu_{2}{ }^{6}+1680 \lambda_{2}{ }^{4} \mu_{2}{ }^{4}+20160 \lambda_{2}{ }^{6} \mu_{2}{ }^{2}+40320 \lambda_{2}{ }^{8}\right)=\mathrm{P}_{1} \frac{\sum_{i=1}^{n} x_{i_{1}}{ }^{8}}{\mathrm{n}_{1}}+\left(1-\mathrm{P}_{1}\right) \frac{\sum_{i=1}^{n} x_{i_{2}}{ }^{8}}{\mathrm{n}_{2}} \text {. }
\end{aligned}
$$

Hence, the parameter estimates using method of moments can be obtained by solving the above equations numerically via $\mathrm{R}$ software.

\section{The Maximum Likelihood Estimation (MLE)}

The maximum likelihood estimation method (MLE) is used to estimate the parameters of the distribution. Now, let $X=\left(x_{1}, x_{2}, \ldots, x_{n}\right)$ is a random sample then the likelihood function of a given distribution is defined as:

$$
L(\theta)=\prod_{i=1}^{n} f\left(x_{i} ; \underline{\theta}\right) .
$$

The maximum likelihood estimates for $\theta$ is calculated by finding a value of $\theta$ that maximizes log-likelihood function. i.e.

$$
\theta=\arg \max \sum_{i=1}^{n} \log f\left(x_{i} ; \underline{\theta}\right) .
$$

For Laplace mixture distribution, define $L(\theta)=L\left(\alpha_{1}, \lambda_{1}, \lambda_{2} \mid \underline{x}\right)$, where the location parameters are known, and equals $\mu_{1}=0, \mu_{1}=2$. Then 


$$
\begin{gathered}
L(\theta)=\prod_{i=1}^{n} f\left(x_{i} ; \alpha_{1}, \lambda_{1}, \lambda_{2}\right)=\prod_{i=1}^{n}\left[\frac{\alpha_{1}}{2 \lambda_{1}} \exp \left(-\frac{\left|x_{i}\right|}{\lambda_{1}}\right)+\frac{\left(1-\alpha_{1}\right)}{2 \lambda_{2}} \exp \left(-\frac{\left|x_{i}-2\right|}{\lambda_{2}}\right)\right] . \\
\log L(\theta)=\sum_{i=1}^{n} \log \left[\frac{\alpha_{1}}{2 \lambda_{1}} \exp \left(-\frac{\left|x_{i}\right|}{\lambda_{1}}\right)+\frac{\alpha_{2}}{2 \lambda_{2}} \exp \left(-\frac{\left|x_{i}-2\right|}{\lambda_{2}}\right)\right] .
\end{gathered}
$$

Next, to find the maximization of log-Likelihood we need to find the derivatives of log-likelihood function with respect to distribution parameters see [10], then we have

$$
\begin{gathered}
\frac{\partial l}{\partial \alpha_{1}}=\sum_{i=1}^{n} \frac{\frac{1}{2 \lambda_{1}} \exp \left(-\frac{\left|x_{i}\right|}{\lambda_{1}}\right)-\frac{1}{2 \lambda_{2}} \exp \left(-\frac{\left|x_{i}-2\right|}{\lambda_{2}}\right)}{\left[\frac{\alpha_{1}}{2 \lambda_{1}} \exp \left(-\frac{\left|x_{i}\right|}{\lambda_{1}}\right)+\frac{\alpha_{2}}{2 \lambda_{2}} \exp \left(-\frac{\left|x_{i}-2\right|}{\lambda_{2} \mid}\right)\right]} . \\
\frac{\partial l}{\partial \lambda_{1}}=\sum_{i=1}^{n} \frac{\exp \left(-\frac{\left|x_{i}\right|}{\lambda_{1}}\right)\left(\alpha_{1}\left|x_{i}\right|-\alpha_{1} \lambda_{1}\right)}{2 \lambda_{1}{ }^{3}\left[\frac{\alpha_{1}}{2 \lambda_{1}} \exp \left(-\frac{\left|x_{i}\right|}{\lambda_{1}}\right)+\frac{\alpha_{2}}{2 \lambda_{2}} \exp \left(-\frac{\left|x_{i}-2\right|}{\lambda_{2}}\right)\right]} . \\
\frac{\partial l}{\partial \lambda_{2}}=\sum_{i=1}^{n} \frac{\exp \left(-\frac{\left|x_{i}-2\right|}{\lambda_{2}}\right)\left(\alpha_{2}\left|x_{i}\right|-\alpha_{2} \lambda_{2}\right)}{2 \lambda_{2}^{3}\left[\frac{\alpha_{1}}{2 \lambda_{1}} \exp \left(-\frac{\left|x_{i}\right|}{\lambda_{1}}\right)+\frac{\alpha_{2}}{2 \lambda_{2}} \exp \left(-\frac{\left|x_{i}-2\right|}{\lambda_{2}}\right)\right]} .
\end{gathered}
$$

The next step is to set the equation (34), equation (35), and equation (36) to zero. Then, solve the system for the three parameters $\alpha_{1}, \lambda_{1}$ and $\lambda_{2}$, this step obtains the MLE of $\alpha_{1}, \lambda_{1}$ and $\lambda_{2}$. In practical, the parameter estimations via MLE method are obtained numerically using Newton-Raphson method by providing initial values for parameters. This is done through the implementation of method using R software

\section{Simulation Study}

We study the effectiveness of Laplace mixture model by providing two scenarios. Firstly, we obtain the MLE estimations for a two components Laplace mixture distribution, and for simplicity, we assume that the location parameters $\mu_{1}=0$ and $\mu_{2}=2$. We study two case studies as illustrated in what follows:

Firstly: Random samples of different sizes 50, 100, 200, 500, 1000, and 1500 are drawn from Laplace mixture distribution with scale parameter equal $\lambda_{1}=\lambda_{2}=1$, and the mixing parameter equals $\alpha_{1}=0.5$. The model parameters are estimated using MLE method, the results are shown in Table 6. This model is iterated 100 times to conduct the consistent of model parameter estimates over 100 iteration, the results are summarized in Table 7 which discussed the properties of each parameter estimates where the location parameters are known. The estimate properties are studied by obtaining the bias, the variance and the mean squared error (MSE) of parameter estimates, we also compute the root mean squared error of parameter estimates. The results show the reasonable MLE estimates for $\alpha_{1}, \lambda_{1}$ and $\lambda_{2}$ and all estimates have good values of Bias, MSE and RMSE. The histogram of this simulation data is displayed in Figure 2.

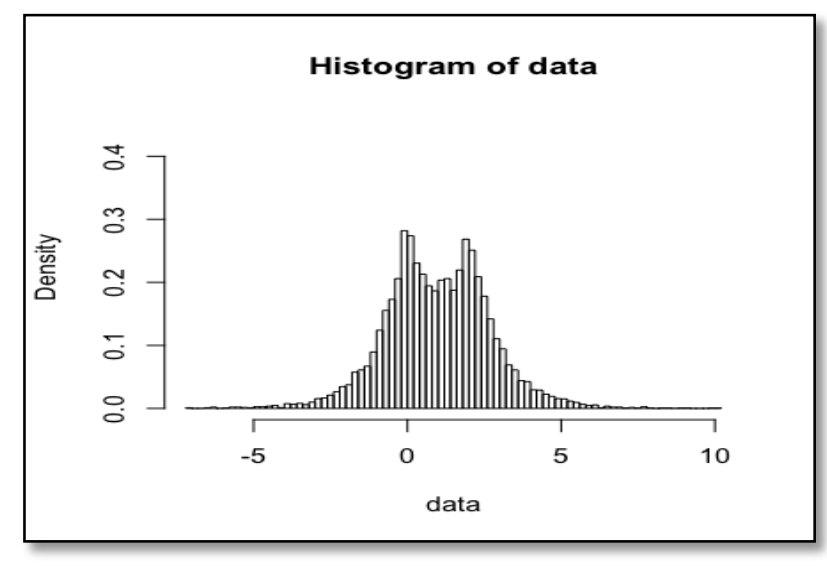

Figure 2. (Histogram of data) Simulation data from Laplace mixture model with $\alpha_{1}=0.5, \mu_{1}=0, \mu_{2}=2$ and $\lambda_{1}=\lambda_{2}=1$

Secondly: We generate a random sample of 1000 data points from Laplace mixture distribution with the same previous assumptions $\left(\alpha_{1,2}=0.5, \mu_{1}=0\right.$ and $\left.\mu_{2}=2\right)$ where scale parameters equal $\lambda_{1}=1$ and $\lambda_{2}=2$. 
Table 6. Simulation study: Estimated parameters for Laplace mixture model using MLE method, where $\alpha_{1}=\alpha_{2}=0.5, \mu_{1}=0, \mu_{2}=2$ and $\lambda_{1}=$ $\lambda_{2}=1$

\begin{tabular}{|c|c|c|c|c|c|}
\hline & & $\widehat{\boldsymbol{\alpha}}_{\mathbf{1}}$ & $\widehat{\boldsymbol{\alpha}}_{\mathbf{2}}$ & $\hat{\boldsymbol{\lambda}}_{\mathbf{1}}$ & $\hat{\boldsymbol{\lambda}}_{\mathbf{2}}$ \\
\hline \multirow{4}{*}{ Sample size } & $\mathbf{5 0}$ & 0.5008221 & 0.4991779 & 0.9535337 & 0.9781209 \\
\cline { 2 - 6 } & $\mathbf{1 0 0}$ & 0.4764069 & 0.5235931 & 0.9286988 & 0.9959736 \\
\cline { 2 - 6 } & $\mathbf{2 0 0}$ & 0.5055666 & 0.4944334 & 1.015432 & 0.9848837 \\
\cline { 2 - 6 } & $\mathbf{5 0 0}$ & 0.5055416 & 0.4944584 & 1.007108 & 0.9894546 \\
\cline { 2 - 6 } & $\mathbf{1 0 0 0}$ & 0.4993363 & 0.5006637 & 1.000324 & 0.9988492 \\
\hline
\end{tabular}

Table 7. The properties of MLE estimated Laplace mixture parameters for the simulated, where $\alpha_{1}=\alpha_{2}=0.5, \mu_{1}=0, \mu_{2}=2$ and $\lambda_{1}=\lambda_{2}=1$

\begin{tabular}{|c|c|c|c|c|}
\hline \multirow{2}{*}{} & \multicolumn{4}{|c|}{ Model parameters } \\
\cline { 2 - 5 } & $\boldsymbol{\alpha}_{\mathbf{1}}$ & $\boldsymbol{\alpha}_{\mathbf{2}}$ & $\boldsymbol{\lambda}_{\mathbf{1}}$ & $\boldsymbol{\lambda}_{\mathbf{2}}$ \\
\hline Initial values & 0.5 & 0.5 & 1 & 0.99651 \\
\hline estimate & 0.501317 & 0.49868 & 0.9989 & 0.003490 \\
\hline Bias & 0.0013168 & 0.001317 & 0.00107 & $3.70 \times 10^{-5}$ \\
\hline Variance & $5.47 \times 10^{-6}$ & $5.47 \times 10^{-6}$ & $5.52 \times 10^{-6}$ & $4.92 \times 10^{-6}$ \\
\hline MSE & $7.21 \times 10^{-6}$ & $7.21 \times 10^{-6}$ & $6.67 \times 10^{-6}$ & $4.92 \times 10^{-5}$ \\
\hline RMSE & $1.44 \times 10^{-5}$ & $1.44 \times 10^{-5}$ & $6.67 \times 10^{-6}$ & $\boldsymbol{L}^{-6}$ \\
\hline
\end{tabular}

Table 8. Simulation study Estimated parameters for Laplace mixture model using MLE method, where $\alpha_{1}=\alpha_{2}=0.5, \mu_{1}=0, \mu_{2}=2, \lambda_{1}=$ 1 and $\lambda_{2}=2$

\begin{tabular}{|c|c|c|c|c|}
\hline \multirow{2}{*}{} & \multicolumn{4}{|c|}{ Model parameters } \\
\cline { 2 - 5 } & $\boldsymbol{\alpha}_{\mathbf{1}}$ & $\boldsymbol{\alpha}_{\mathbf{2}}$ & $\boldsymbol{\lambda}_{\mathbf{1}}$ & $\boldsymbol{\lambda}_{\mathbf{2}}$ \\
\hline Initial values & 0.5 & 0.5 & 1 & 2.059199 \\
\hline estimate & 0.541058 & 0.458942 & 1.090983 & 0.059199 \\
\hline Bias & 0.041057 & 0.041057 & 0.090983 & 0.183973 \\
\hline Variance & 0.001050 & 0.001050 & 0.067408 & 0.003504 \\
\hline MSE & 0.001686 & 0.001686 & 0.008278 & 0.001752 \\
\hline
\end{tabular}

\section{Conclusions}

This paper proposed Laplace mixture distribution with two components. The properties of the proposed mixture model are discussed theoretically. Moreover, the parameters are estimated using method of moments and maximum likelihood estimations method. The simulation study has been indicated that the model parameter estimates provide reasonable results and close to true values of model parameters. For future work, one can apply semi-parametric methods to derive parameter estimations for Laplace mixture distribution with $k$ components.

\section{Acknowledgements}

The second author is grateful to King Abdulaziz University and Al-Jouf University for the constructive cooperation between them. This article is also a part of the master's thesis of the second author, supervised by Dr. Zakiah Kalantan.

\section{REFERENCES}

[1] E. Amini-Seresht, Y. Zhang. Stochastic comparisons on two finite mixture models. Operations Research Letters, 45(5), 475-480, 2017.

[2] D. Bhowmick, A. Davison, D. R. Goldstein, Y. Ruffieux. A Laplace mixture model for identification of differential expression in microarray experiments: Biostatistics, 7(4), 630-641, 2006.

[3] M. A. Figueiredo, A. K. Jain. Unsupervised learning of finite mixture models: IEEE Transactions on pattern analysis and machine intelligence, 24(3), 381-396, 2002.

[4] S. Kotz, T. Kozubowski, K. Podgorski. The Laplace distribution and generalizations: a revisit with applications to communications, economics, engineering, and finance, Springer Science \& Business Media, 2012.

[5] M. Ali, S. Nadarajah. Information matrices for normal and Laplace mixtures: Information Sciences, 177(3), 947-955, 2007.

[6] G. McLachlan, D. Peel. Finite Mixture Models. John Wiley and Sons, Inc., New York. MR17894, 2000.

[7] K. Pearson. Contribution to the mathematical theory of 
evolution. Philosophical Transactions of the Royal Society, 185, 71-110, 1894.

[8] J. Philip, Davis \& R. Philip. Methods of numerical integration. Courier Corporation, 0486453391, 2007.

[9] DV Ramana Murty, G. Arti, M, Vivekananda Murty. Two component mixture of Laplace and Laplace type distributions with applications to manpower planning models. International Journal of Statistics and Applied Mathematics, 3(4), 01-11, 2018.

[10] R. M. Norton. The Double Exponential Distribution: Using Calculus to Find a Maximum Likelihood Estimator. The American Statistician, 38 (2), 135-136, 1984.

[11] S. Shenoy, D. Gorinevsky. Gaussian-Laplace mixture model for electricity market. Paper presented at the Decision and Control (CDC), 2014 IEEE 53rd Annual Conference on, 2014.

\footnotetext{
i Conference Papers
}

Zakiah Kalantan, Faten Alrewely, A 2-Components Laplace Mixture Model: Properties and Parametric Estimations, Conference: The 4 International Conference on Computing, Mathematics and Statistics 2019 (iCMS2019). At: Ombak Villa, Langkawi Island, Malaysia. 